\title{
A New Optimization Approach Using Smoothed Images Based on ACO for Medical Image Registration
}

\author{
Sunanda Gupta \\ Manav Rachna International University /ECE, Faridabad, 121004, India \\ E-mail: sunanda.fet@mriu.edu.in \\ Naresh Grover \\ Manav Rachna International University (MRIU)/ECE, Faridabad, 121004, India \\ E-mail: dean.academics@mriu.edu.in \\ Zaheeruddin \\ Jamia Millia Islamia (A Central University)/ Electrical Engineering, New Delhi, 110025, India \\ E-mail: zaheeruddin@jmi.ac.in
}

\begin{abstract}
This paper studies on image registration using Ant Colony Optimization technique of the medical images. Ant Colony Optimization algorithm has ability of global optimization and facilitates quick search of optimal parameters for image registration. In this paper, a modified Ant Colony Optimization algorithm on preprocessed images is proposed to improve the accuracy in terms of PSNR (peak signal to noise ratio), Entropy and convergence speed. Preprocessing of images is adopted to remove noise so that extracted features provide more accurate and precise information about the image and results are more suitable for further analysis. The experimental results demonstrate the performance of proposed methodology as compared with traditional approaches as very promising.
\end{abstract}

Index Terms-Medical imaging, image registration, ACO, Segmentation, Gaussian filter.

\section{INTRODUCTION}

Swarm intelligence research is originated from progress work into the simulation of emergence of collective intelligence behavior of real ants. Ants have unusual intelligence to discover efficient solutions for shortest path problems between the nest and food source, on their way back from food source by a trail of a substance, called - a pheromone. Depending upon this pheromone level communication, the shortest path with greatest density of pheromone level is considered and the ants will tend to follow the path with more pheromone [1]

M. Dorigo (1992) was first to use this approach to solve the travelling salesman problem [2]. This algorithm is referred to as Ant colony optimization algorithm (ACO). After that ACO has gained widespread success in solving diverse optimization problems, such as the Justin-Time (JIT) sequencing problem with multiple objectives [3], a machine tool tardiness problem [4], the vehicle routing problem [5] and many more.

Image registration plays a vital role in medical imaging. It is a process of aligning two images namely reference and target images of the same scene. Reference image is the main image for which the registration is to be applied. Target image is the image on which the registration is applied, so that the target image can be rotated at some angle or some other effects etc. It can be used in medical applications to match images of same patient acquired over a period of time. Fig. 1 represents the steps involved in a image registration process [6].

Now-a-days, image registration is also being applied in image guided surgery interventions, to monitor the progress of diseases and healing therapy in serial imaging analysis. Generally, image registration methods can be classified as feature based [7], gradient based [8, 9] and area based [10,11]. Many hybrid approaches [12, 13, 14] of image registration are also possible. Dhawan, (2012) suggests an integrated approach based on rigid \& nonrigid deformation for image registration in case of complete and deformed data set[15].

An approach of image registration based on features using ACO on preprocessed images is presented in this paper. In feature-based image registration methods, correspondence is established between features also known as characteristics or control points like corners, edges, closed-boundary regions, line intersections and contours etc. extracted in the float image and those extracted in the reference image. Variety of feature descriptors using spatial association and similarity measures are used for this purpose. Most importantly, extracted features are also considered as invariant to image deformation. Mostly feature based methods show image registration relatively fast, but lacks in feature extraction robustness and feature matching accuracy [16].

In this paper, modified ACO algorithm is applied on noise free images to obtain segmented images to have sufficient volume control points automatically and to calculate features' parameters for image registration. 


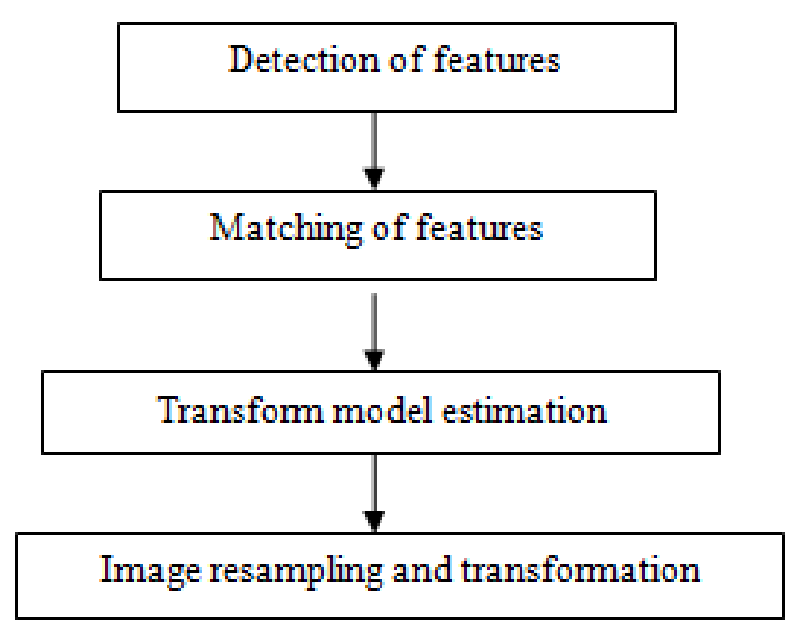

Fig.1. Steps Involved in Image Registration

The rest of the paper is organized as follows: Section II deals with related works done in this field. Section III contains the smoothing process for the images used in image registration. Section IV describes ACO (Ant Colony Optimization) approach to extract the features' parameters; Results for performance measures for proposed methodology are discussed in Section V. Conclusion and Future Scope are presented in Section VI.

\section{RELATED WORK}

Image registration is usually used to extract complementary information of images acquired from different modalities [17] and to examine images of same modality accurately $[18,19]$. In medical imaging, image registration enables to locate and estimate subtle changes that appear during acquisition of consecutive images. Many papers based on different image registration techniques like mutual information and genetic algorithm etc. have been studied while completing this work. Maes F. (2003) and Kishankumar M. (2014) proposed mutual information method to measure the information redundancy [20, 21]. Kostelec P.J. (2003) proposed an introduction to image registration applied in medical imaging which is able to spot difference between two images and can be used for medical application later [22]. Andrea (2012), Pandey et.al. (2012) gave new intensity-based image registration (IR) approaches based on a modern, real-coded genetic optimization algorithm [23, 24]. A novel continuous medical image registration (CMIR), fuzzy logic based approach was proposed by Moulick (2013) [25].

\section{SMOOTHING}

Feature appearance is enhanced using some preprocessing steps like gamma correction and gradient methods due to the noise involved in medical images. Particularly, the accuracy of registration algorithm rely on the quality of procedure opted to obtain segmented images (later used to extract features) in previous step for features matching [12].

Smoothing is done in order to make the image noise free so that maximum amount of information is extracted from the image that results in better feature extraction process. Many filters are used for smoothing. In this proposed approach Gaussian filter is used and the image is first converted into frequency domain by applying FFT and then Gaussian filter is applied on this converted image, for smoothing process. A threshold is set and the components that are out of the frequency threshold are rescaled, with the help of which the noise is removed. The output image is smoothed image with less noise and is used for the registration purpose further. Main reason to prefer Gaussian filter is that mostly the noise that affects the data is Gaussian noise, so this is better for such type of noise

\section{ACO (ANT COLONY OptimizATiON)}

ACO was first introduced by M. Dorigo in 1992. One basic idea of the ACO approach is to use the counterpart of the pheromone trail used by real ants as a medium for communication and as an indirect form of memory of previously found solutions.

Ant colony optimization is a nature-inspired optimization algorithm inspired by food foraging behavior exhibited by ant societies [26, 27]. Through some biologist's point of view, the visual sensory organs of the real world ants are rudimentary by nature. The ants communicate using a chemical substance called pheromone. In journey of an ant, it accumulates a constant amount of pheromone that other ants can follow and if it came after the trail, the ant's own pheromone reinforces the current trail, the growth in pheromone increases the probability of the next ant selecting the path. Therefore, the more the ants travel on a path, the more attractive the path becomes for consecutive ants. Over time, as more ants are capable to complete the shorter route. Therefore on shorter paths pheromone densities stay high because pheromone is laid down faster and the longer paths are less reinforced and finally abandoned [28]. This method of positive feedback eventually leads the ants to follow the smaller paths. It is this usual experience that encouraged the development of the ACO meta-heuristic.

Using this kind of method, the main advantage is that, it extracts remarkable features of the image, greatly compresses the image information, and has good robustness, small amount of calculation, fast speed. It only needs four processes including initialization process, construction process, update process and decision process. Fig. 2 represents the steps involved for ACO optimization.

Hill in 1993 proposed a feature space that deployed the combination of corresponding pixels intensities in a set of two images. This feature space alters with the variations in the alignment of the images. If the images are accurately registered, then specific groups for corresponding anatomical structures in feature space could be recognized. In case of non-registration, the structures of reference image do not match to the corresponding pixels in the target image and effects dispersion. This property of the feature space permits the use of dispersion as a image registration measure [29]. 
Later Collignon \& Studholme demonstated entropy as a registration measure in 1995. After this P. Voila \& Wells proposed mutual information as a new measure for image registration in 1997 and became one of the important techniques of image registration [30].

Mutual information can be represented in many ways. Mutual information (MI) in terms of Entropy is given as:

$$
M I=H_{a}+H_{b}-H_{a b}
$$

where

$\mathrm{H}_{\mathrm{a}}$ is Shanon's entropy of reference image

$\mathrm{H}_{\mathrm{b}}$ is Shanon's entropy of target image

$\mathrm{H}_{\mathrm{ab}}$ shows joint entropy of reference and target image

Amount of pheromone updated is given as:-

$$
\tau_{i j}=(1-\rho) \tau_{i j}+\Delta \tau_{i j}
$$

$\tau_{\mathrm{ij}}$ is the amount of pheromone on edge (i,j) $\rho$ rate of pheromone evaporation

$\Delta \tau_{\mathrm{ij}}$ is the amount of pheromone deposited

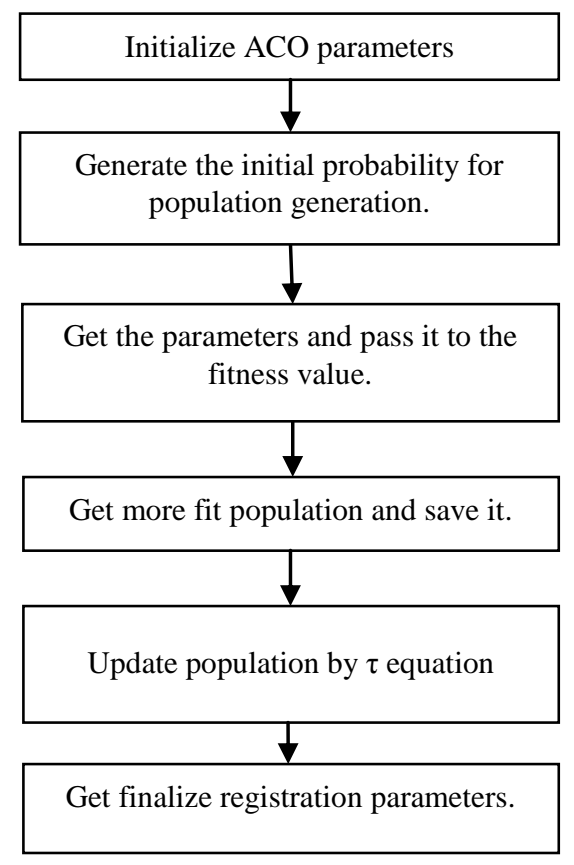

Fig.2. Steps for ACO Optimization

\section{A. Proposed Methodology}

In an ACO based optimization method, the design of the pheromone update strategy, and the measurement of the quality of the solutions are critical. This technique comprises of two types of images. one image is called reference image and other is called the target image.

Fig. 3 depicts the steps of proposed methodology being followed.

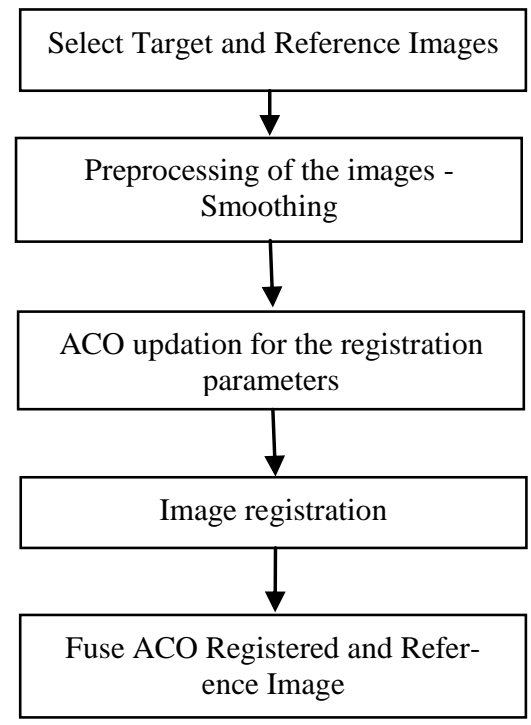

Fig.3. Steps for Proposed Methodology

The steps listed below are being followed while implementing this algorithm in MATLAB for medical image registration using ACO.

Step 1. Choose the reference image and Target image.

Step 2. Under the preprocessing step, both the images (reference \& target images) are converted into frequency domain through FFT. These images are further passed through the Gaussian filter to remove noise to get smoothed reference image and target image. Then these images are converted back into spatial domain using IFFT.

Step 3. First of all the parameters of the ACO are initialized. The parameters that are obtained are $\mathrm{X} \& \mathrm{Y}$ co-ordinates, and angle calculated by using equation (3)

$$
\tau_{i j} \leftarrow(1-\rho) \tau_{i j}+\sum_{k=1}^{m} \Delta \tau_{i j}^{k}
$$

Step 4. For the population generation generate the initial probablity equation given below:-

$$
\rho_{i j}=\frac{\left(\tau_{i j}^{\alpha}\right)\left(\eta_{i j}^{\beta}\right)}{\sum\left(\tau_{i j}^{\alpha}\right)\left(\eta_{i j}^{\beta}\right)}
$$

$\alpha$ is a parameter to control the influence of $\tau_{\mathrm{ij}}$. $n_{\mathrm{ij}}$ is the desirability of edge $(\mathrm{i}, \mathrm{j})$.

$\beta$ is a parameter to control the influence of $n_{\mathrm{ij}}$.

Step 5. After getting the parameters, pass them to the fitness value.

Using the entropy MI (Mutual information ) equation given below

$$
M I=H_{a}+H_{b}-H_{a b},
$$


Step 6. Get the best population out of the population generated by the following equation

$$
\tau_{i j} \leftarrow(1-\rho) \cdot \tau_{i j}+\rho \cdot \Delta \tau_{i j}^{b e s t}
$$

And finally saves the results.

Step 7. Now, update the population that is saved by the equation (7) given below.

$$
\tau_{i j}=(1-\rho) \tau_{i j}+\Delta \tau_{i j}
$$

Step 8. Finally, get the registration parameters for the registration of the images i.e. $\mathrm{X}, \mathrm{Y} \&$ angles.

\section{RESULTS AND DISCUSSION}

In this paper, image registration based on this algorithm on two types of images (MRI, MRI_1) using Matlab R2013b is performed. The performance, efficiency and robustness of presented algorithm is demonstrated by extensive image registration experiments. Experiments are performed for different angle of rotation. Figs.4-7 show results of medical image registration for different angle of rotations.

Figs. 4, 5 show results of medical image registration of image MRI_1 at rotation angle of $-3,-5$ degree. Similarly results of images MRI is shown in figs. $6 \& 7$ for different angles of rotation and are tabulated in table 1 and table2 for each image implementations respectively. Figs. 8 \& 9 show the correlation, time for registration, S/N (signal to noise) ratio, mutual information for both images ( MRI, MRI_1) respectively.

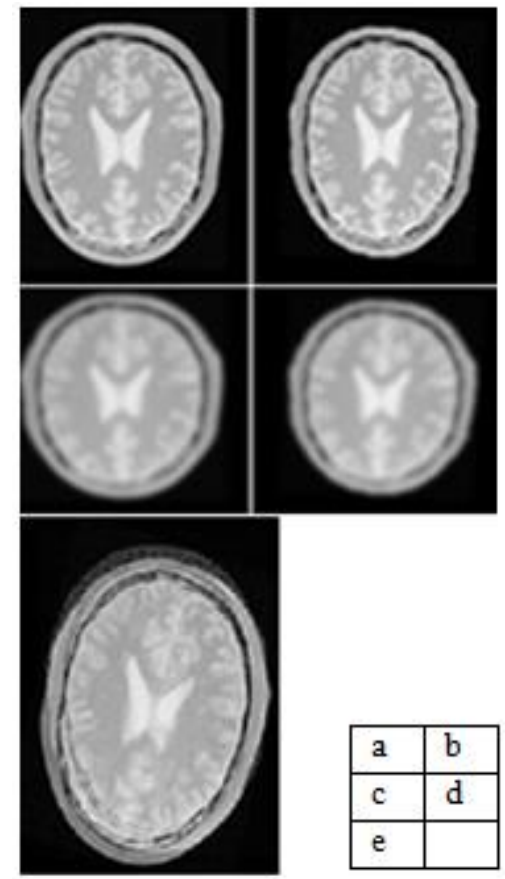

Fig.4. Image MRI_1 with angle of rotation -3 degree (a) Reference image, (b) Target image (c) Preprocessed Reference image (d) Preprocessed target image (e) ACO Registered image
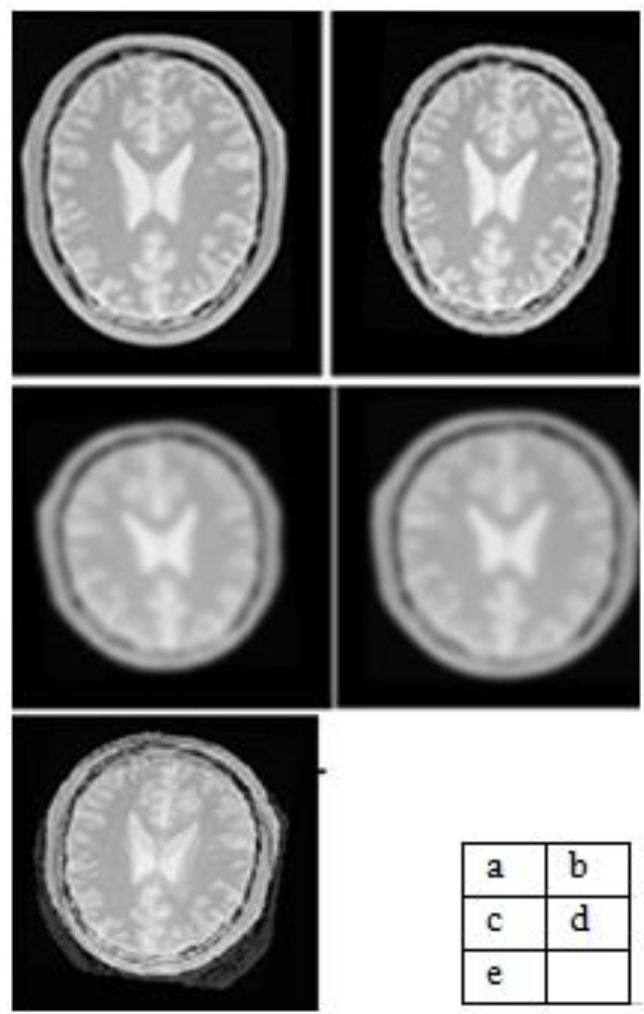

\begin{tabular}{|l|l|}
\hline$a$ & $b$ \\
\hline$c$ & $d$ \\
\hline$e$ & \\
\hline
\end{tabular}

Fig.5. Image MRI_1 with angle of rotation -5 degree (a) Reference image, (b) Target image (c) Preprocessed Reference image (d) Preprocessed target image (e) ACO Registered image
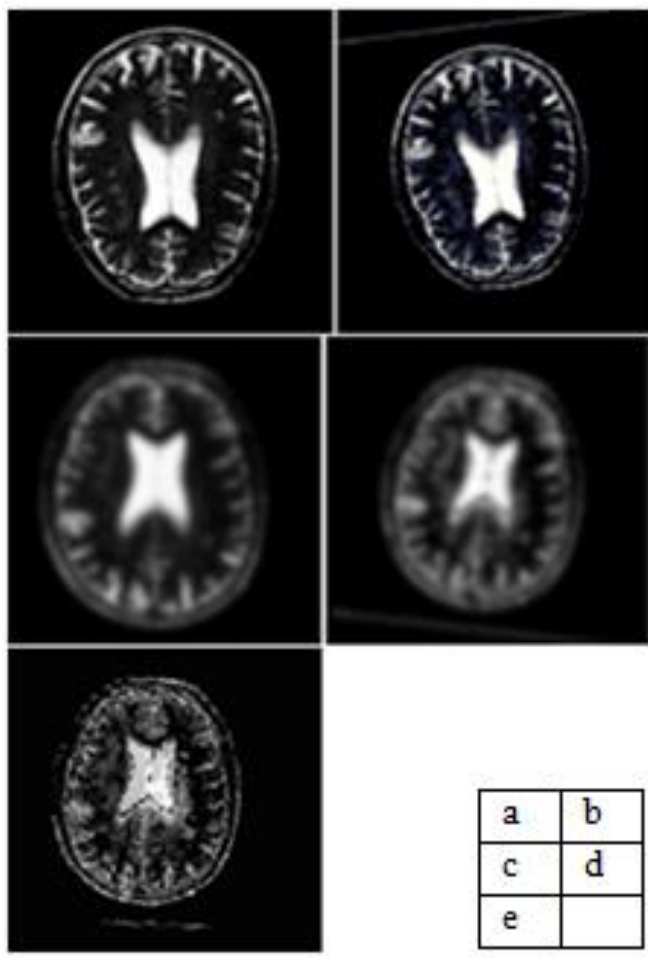

Fig.6. Image MRI with angle of rotation -7 degree (a) Reference image, (b) Target image (c) Preprocessed Reference image (d) Preprocessed target image (e) ACO Registered image 


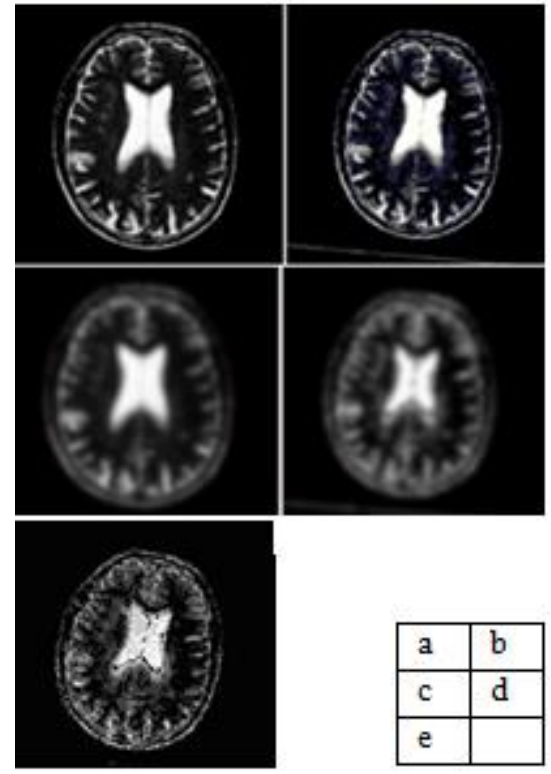

Fig.7. Image MRI with angle of rotation -5 degree (a) Reference image, (b) Target image (c) Preprocessed Reference image (d) Preprocessed target image (e) ACO Registered image

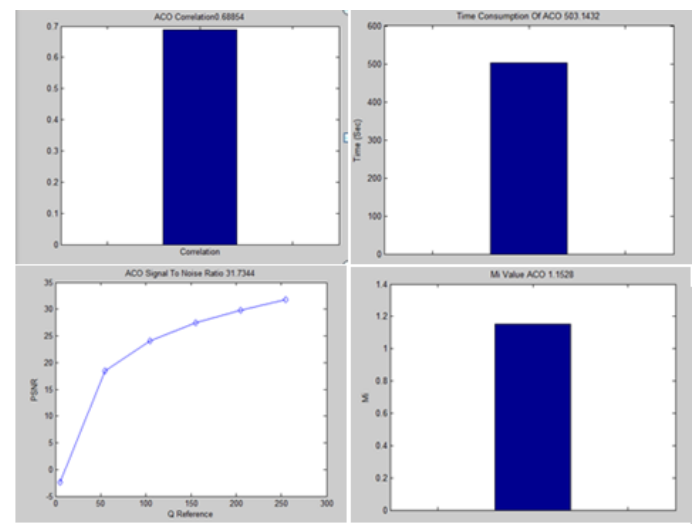

\begin{tabular}{|l|l|}
\hline $\mathrm{a}$ & $\mathrm{b}$ \\
\hline $\mathrm{c}$ & $\mathrm{d}$ \\
\hline
\end{tabular}

Fig.8. Image MRI with angle of rotation -5 degree (a) Correlation (b) Time Consumption (c) Signal to Noise ratio (d) Mutual Information

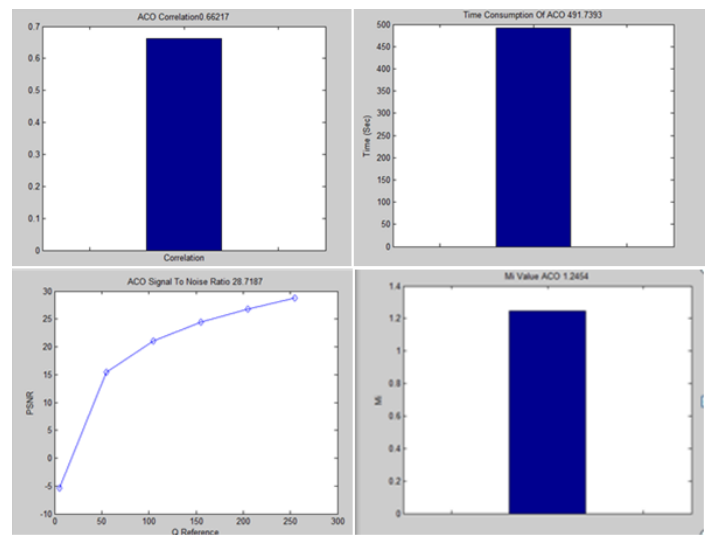

\begin{tabular}{|l|l|}
\hline $\mathrm{a}$ & $\mathrm{b}$ \\
\hline $\mathrm{c}$ & $\mathrm{d}$ \\
\hline
\end{tabular}

Fig.9. Image MRI_1 with angle of rotation -3 degree (a) Correlation (b) Time Consumption (c) Signal to Noise ratio (d) Mutual Information
Table 1. Medical Image Registration of Image MRI_1 for Different Rotation Angles

\begin{tabular}{|c|c|c|c|c|c|c|}
\hline \multicolumn{7}{|c|}{ Image MRI_1 } \\
\hline $\begin{array}{c}\text { Rotation } \\
\text { angle }\end{array}$ & $\theta$ & $\begin{array}{c}\text { Corre- } \\
\text { lation }\end{array}$ & $\begin{array}{c}\text { Time } \\
(\mathrm{sec})\end{array}$ & $\begin{array}{c}\text { Mutual } \\
\text { information }\end{array}$ & $\begin{array}{c}\text { S/N } \\
\text { ratio }\end{array}$ & MSE \\
\hline-3 & -2.9 & 0.66 & 491.7 & 1.25 & 28.71 & 37.34 \\
\hline-5 & -5.13 & 0.84 & 523.0 & 1.41 & 32.14 & 39.72 \\
\hline-7 & -6.7 & 0.85 & 517.4 & 1.73 & 32.45 & 36.93 \\
\hline
\end{tabular}

Table 2. Medical Image Registration of Image MRI for Different Rotation Angles

\begin{tabular}{|c|c|c|c|c|c|c|}
\hline \multicolumn{7}{|c|}{ Image MRI } \\
\hline $\begin{array}{c}\text { Rotation } \\
\text { angle }\end{array}$ & $\theta$ & $\begin{array}{c}\text { Corre- } \\
\text { lation }\end{array}$ & $\begin{array}{c}\text { Time } \\
(\mathrm{sec})\end{array}$ & $\begin{array}{c}\text { Mutual } \\
\text { information }\end{array}$ & $\begin{array}{c}\text { S/N } \\
\text { ratio }\end{array}$ & MSE \\
\hline-3 & -3.1 & 0.69 & 496.4 & 0.96 & 31.5 & 45.88 \\
\hline-5 & -4.9 & 0.69 & 503.1 & 1.15 & 31.73 & 43.61 \\
\hline-7 & -7.4 & 0.54 & 502.2 & 0.86 & 31.77 & 43.20 \\
\hline
\end{tabular}

The parameters which are considered for the results during implementation are processing time, Mutual information and the angle of rotation at which the target image is to change so as to get the registered image. Finally the image obtained best from the ACO using this information is registered with the reference image. Experiments on medical images also show better efficiency, time consumption, correlation \& mutual information (entropy) of the proposed algorithm as compared to many standard methods of the literature.

\section{CONCLUSION AND FUTURE SCOPE}

In this work, a new approach of medical image registration based on ACO on smoothed images for image segmentation and for extracting points' features is proposed. Results are obtained for different rotation angles. It is inferred that proposed algorithm shows good registration when float image is rotated at an angle. Also, the proposed registration algorithm is robust against different image transformations like standard SIFT, other recent fuzzy and neural based methods quantitatively.

Here registration is implemented for single modality image registration and needs to be extended and tested for multimodality image registration. The proposed registration algorithm also needs to be tested for image registration in case of scaled float image.

The performance, efficiency and robustness of presented algorithm is demonstrated by extensive image registration experiments for different conditions. Our experimental results show that the presented algorithm is very suitable for medical image registration for different rotation angles. Experiments on medical images also show efficiency in terms of accuracy of the proposed algorithm as compared to many standard methods of the literature.

\section{REFERENCES}

[1] Wen Peng, Ruofeng Tong, Guiping Qian, Jinxiang Dong, "A Constrained Ant Colony Algorithm for Image Registration " Computational Intelligence and Bioinformatics Volume 4115 of the series Lecture Notes in Computer Science pp 1-11. 
[2] Dorigo, M. (1992), "Optimization, Learning and Natural Algorithms". Ph.D. Thesis, Italy.

[3] McMullen, P.R., 'An Ant Colony Optimization Approach to Addressing a JIT Sequencing Problem with Multiple Objectives'. Artificial Intelligence, 309-317 (2001).

[4] Bauer, A., Bullnheimer, B., Hartl, R.F., 'An Ant Colony Optimization Approach for the Single Machine Tool Tardiness Problem'. In: Proceeding of the Congress on Evolutionary Computation, pp. 1445-1450 (1999).

[5] Bullnheimer, B., Hartl, R.F., Strauss, C., (1997)," Applying the Ant System to the Vehicle Routing Problem". In: The Second Metaheuristics International Conference, France.

[6] Gupta,S., chakarvarti,S.K. \& Zaheeridin (2013), "Image Registration Methods: A Short Review ", Columbia International Publishing American Journal of Algorithms and Computing (2013) Vol. 1 No. 1 pp. 39-49.

[7] Sharma, N., Ray, A.K., Sharma, S., Shukla, K.K., Pradhan, S. and Aggarwal, L.M. (2008), "Segmentation and classification of medical images using texture-primitive features: Application of BAM-type artificial neural network", J. Med. Phys. 33, pp. 119-126.

[8] De-Nigris, D., Collins, DL. and Arbel, T.( 2012 ),'Multimodal image registration based on gradient orientations of minimal uncertainty', IEEE Transactions on Medical Imaging, Vol. 31, pp. 2343 - 2354

[9] De-Nigris, D., Collins, DL. and Arbel, T. (2013),"Fast rigid registration of pre-operative magnetic resonance images to intra-operative ultrasound for neurosurgery based on high confidence gradient orientations", International Journal of Computer Assisted Radiology and Surgery, pp.649-61.

[10] Joglekar, Jyoti and Shirish, S. (2012) 'Area Based Image Matching Methods: A Survey', International Journal of Emerging Technology and Advanced Engineering ISSN 2250-2459, Vol. 2, issue1.

[11] Roy, Sujoy (2010) ' Region- based image registration for mosaicking ', International Journal of Computer Applications in Technology, Vol. 37, No.1 pp. 59 - 73.

[12] Gupta,S., Chakarvarti, S.K. \& Zaheeridin (2016), "Medical image registration based on Fuzzy c-means clustering segmentation approach using SURF", Int. J. Biomedical Engineering and Technology, Vol. 20, No. 1, pp.33-50.

[13] Cifor, A., Risser, L., Chung, D., Anderson, EM. and Schnabel, JA. (2013) 'Hybrid feature-based diffeomorphic registration for tumor tracking in 2-D liver ultrasound images' IEEE Transactions on Medical Imaging, pp.1647-56.

[14] Farnia, P., Ahmadian, A., Shabanian, T., Serej, ND and Alirezaie, J. (2014) 'A hybrid method for non-rigid registration of intra-operative ultrasound images with preoperative MR images', international conference Proceedings IEEE in Engineering in Medicine and Biology Society (EMBC), pp.5562-65.

[15] Dhawan, Atam P. (2012) 'Rigid and non- rigid medical image registration methods', International Journal of Biomedical Engineering and Technology Vol. 8, No.2/3 pp. $200-219$.

[16] Mahmoud, Hassan, Masulli, Francesco and Rovetta, Stefano (2013) 'Feature-Based Medical Image Registration Using a Fuzzy Clustering Segmentation Approach', CIBB LNBI 7845, pp. 37-47, Springer-Verlag Berlin Heidelberg.

[17] Peter, Dinesh J., Govindan ,V.K. and Mathew, Abraham T. (2009) 'A robust affine parameter estimation using acquainted non- convexity multiresolution based registration', International Journal of Computer Aided Engineer- ing and Technology, Vol. 1, No.4 pp. 455 - 479.

[18] Maintz, J.B.A. and Viergever, M.A. (1998) 'A survey of Medical Image Registration', Medical Image Analysis, Vol. 2, pp.1-36.

[19] Hallpike, L. and Hawkes, D.J. (2002) 'Medical image registration: an overview', Imaging, Vol. 14, 455-463.

[20] Maes, F., Vandermeulen, D. \& Suetens, P. (2003), "Medical imaging registration using mutual information" proceedings of the IEEE, vol. 91, issue. 10, 1699-1722.

[21] Kishan kumar, M. \& Patel, M., (2014), "Medial Image Registration Based on Information Theoretic Approach". International Journal of Advanced Engineering and Nano Technology (IJAENT), Volume-1, Issue-6, pp 24-26.

[22] Kostelec P. j. \& Periaswamy, S., (2003) "Image Registration for MRI". Modern Signal Processing Publications (MSRI), Volume 46, pp161-184.

[23] Andrea Valsecchi, Damas, S. \& Santamaria, J., (2012) "An Image Registration Approach using Genetic Algorithms" WCCI IEEE World Congress on Computational Intelligence June, 10-15, pp. 416-423.

[24] Pandey, N. K., Gupta, A., \& Mishra, A. K., Sharma, S. (2012), "Image registration: An application of image processing", International Journal of Modern Engineering Research (IJMER) Vol. 2, Issue. 5, pp-3271-3274.

[25] Moulick, H.N. \& Chatterjee, A., (2013) "Biomedical Image Registration Using Fuzzy Logic" International Journal of Computer Trends and Technology (IJCTT) - volume 4 Issue 5, pp 1394-1406.

[26] M. Dorigo and S. Thomas, "Ant Colony Optimization". Cambridge: MIT Press, 2004.

[27] H.B. Duan, (2005) "Ant Colony Algorithms: Theory and Applications". Beijing: Science Press.

[28] M. Dorigo, M. Birattari and T. Stutzle, (2006) "Ant colony optimization", in proc. of the IEEE Computational Intelligence Magazine, pp.28.39.

[29] Kosiński, W., Michalak, P., Gut, P., (2012) "Robust Image Registration Based on Mutual Information measure", Journal of Signal and Information Processing, 3, 175-178.

[30] P. Viola and W. M. Wells III, (1997) "Alignment by Maximiza- tion of Mutual Information," International Journal of Com- puter Vision, Vol. 24, No. 2, pp. 137-154.

\section{Authors' Profiles}

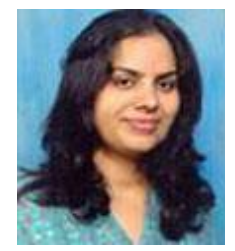

Sunanda Gupta, an assistant professor in ECE department is pursuing her Ph.D from Manav Rachna International university. She received M.Tech Degree in Instrumentation \& Control from M.D.University, Rohtak (Haryana) in the year 2006, and B.E(Honors) Degree in ECE from Institution of Engineers, Calcutta, India in 2000. Her interest includes Medical Image Processing, Neural Network, Fuzzy Logic Control. She is a life member of ISTE \& Associate member of 'The Institution of Engineers', Calcutta, India.

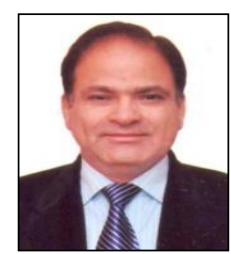

Dr. Naresh Grover did his B.Sc (Engg.) in 1984 and M.Tech in Electronics and Communication Engineering in 1998 from REC Kurukshetra (Now NIT Kurukshetra) and thereafter completed his $\mathrm{PhD}$ from Manav Rachna International University (MRIU). $\mathrm{He}$ has a rich experience of 31 years in academics. He has authored two books on Microprocessors and 
is a co-author for a book on Electronic Components and Materials. His core area of interest is Microprocessors and Digital System Design. Presently he is Dean-Academics at MRIU

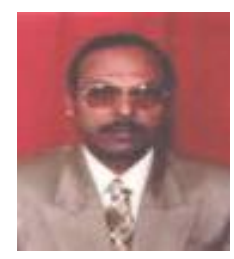

Dr. Zaheeruddin is Professor in the Department of Electrical Engineering, Faculty of Engineering \& Technology, Jamia Millia Islamia (A Central University), New Delhi, India since 2003. He received the B. Sc. Engg. Degree in Electrical and M. Sc. Engg. Degree in Electronics \& Communication from Aligarh Muslim University (AMU), Aligarh (UP) in 1982 and 1988 respectively, and a Ph. D. Degree in Computer Science from Jawaharlal Nehru University (JNU), New Delhi in 2002. He received Indian National Science Academy (INSA) Visiting Fellowship Award for the year 2004-05 to work with Prof. N. R. Pal in Computational Intelligence Lab. at Indian Statistical Institute Kolkata, India. He is a Fellow of IETE (India) and Life Member of World Federation of Soft Computing (U.S.A.), The Institution of Engineers (IE, India), and many more.

How to cite this paper: Sunanda Gupta, Naresh Grover, Zaheeruddin,"A New Optimization Approach Using Smoothed Images Based on ACO for Medical Image Registration", International Journal of Information Engineering and Electronic Business(IJIEEB), Vol.8, No.2, pp.30-36, 2016. DOI: 10.5815/ijieeb.2016.02.04 